\title{
On the Construction of Kernel-Based Adaptive Particle Methods in Numerical Flow Simulation
}

Armin Iske

\begin{abstract}
This contribution discusses the construction of kernel-based adaptive particle methods for numerical flow simulation, where the finite volume particle method (FVPM) is used as a prototype. In the FVPM, scattered data approximation algorithms are required in the recovery step of the WENO reconstruction. We first show how kernel-based approximation schemes can be used in the recovery step of particle methods, where we give preference to the radial polyharmonic spline kernel. Then we discuss important aspects concerning the numerical stability and approximation behaviour of polyharmonic splines. Moreover, we propose customized coarsening and refinement rules for the adaptive resampling of the particles. Supporting numerical examples and comparisons with other radial kernels are provided.
\end{abstract}

\section{Introduction}

The numerical simulation of multiscale phenomena in time-dependent evolution processes is of great importance in many relevant applications from science and technology, which, moreover, incorporates many challenging issues concerning the design of suitable computational methods. Efficient, robust and accurate computer simulations require customized multiscale approximation algorithms, where adaptivity plays a key role.

Particle models have provided very flexible discretization schemes for the numerical simulation of multiscale phenomena in various relevant applications from computational science and engineering. In the modelling of time-dependent evolution processes, for instance, particle models are particularly well-suited to cope with rapid variation of domain geometries and anisotropic large-scale deformations.

Numerical flow simulation by particle methods works with a finite set of scattered particles, where some specific physical properties are attached to the individual par-

Armin Iske

Department of Mathematics, University of Hamburg, Germany e-mail: iske@ math.uni-hamburg.de 
ticles. In numerical simulations of time-dependent evolution processes, the particles are subject to dynamic modifications during the simulation. This requires both customized adaption rules for the adaptive modification of the active particle set, and a suitable strategy for the resampling of the particle values.

Particle flow simulations essentially require powerful approximation algorithms for local scattered data reconstruction. To this end, we prefer to work with kernelbased scattered data approximation by polyharmonic splines. Supporting arguments in favour of this particular meshfree reconstruction scheme are given in this paper, where we address relevant numerical aspects concerning their computational efficiency and flexibility, their optimality, their numerical stability, their approximation behaviour as well as their utility for adaptive concepts of particle flow simulation.

The outline of this article is as follows. In the following Section 2, we briefly review basic facts concerning hyperbolic conservation laws, being the governing equations for the flow simulation model problems that we wish to address. This then leads us to the Eulerian finite volume particle method (FVPM), which we explain in Section 3, followed by a short discussion on the required WENO reconstruction in Section 4. Then, in Section 5, we explain how to apply kernel-based reconstructions in particle flow simulations. This leads us to polyharmonic splines, whose basic features are first explained in Section 6, before we turn to a more detailed discussion concerning their numerical properties in Section 7, where we show that polyharmonic splines provide numerically stable reconstructions at arbitrary local approximation order, unlike other radial kernels. Thereby, we can give a strong recommendation in favour of polyharmonic splines, especially for their application to particle flow simulation. The construction of customized adaption rules for the resampling of the particles is explained in Section 8. Finally, a relevant test case scenario from oil reservoir simulation, the five-spot problem, shows the utility of the proposed kernel-based adaptive particle method in Section 9.

\section{Hyperbolic Conservation Laws}

Numerical flow simulation requires suitable approximation algorithms for the solution of time-dependent hyperbolic conservation laws

$$
\frac{\partial u}{\partial t}+\nabla f(u)=0
$$

where for some domain $\Omega \subset \mathbb{R}^{d}, d \geq 1$, and a compact time interval $[0, T], T>0$, the solution $u:[0, T] \times \Omega \rightarrow \mathbb{R}$ of (1) is sought.

In this problem, $f(u)=\left(f_{1}(u), \ldots, f_{d}(u)\right)^{T}$ denotes a given flux tensor, and it is usually assumed that initial conditions

$$
u(0, x)=u_{0}(x) \quad \text { for } x \in \Omega
$$

at time $t=0$ are given. 
In the relevant nonlinear case, a nonlinear flux $f$ usually leads to discontinuities in the solution $u$, shocks, as observed in many relevant applications, such as fluid flow and gas dynamics. Such discontinuities of the solution $u$ in (1) can easily develop spontaneously even from smooth initial data $u_{0}$ in (2).

Therefore, nonlinear flow simulation requires more sophisticated and flexible mathematical and computational methods to numerically solve the Cauchy problem (1), (2). For a comprehensive introduction to numerical methods for hyperbolic problems we recommend the textbook [16].

\section{Finite Volume Particle Method (FVPM)}

In this section, we explain the main ingredients of the finite volume particle method (FVPM) [10], which we use as a prototype for an Eulerian particle-based concept in numerical flow simulation. In previous work [13], we have also introduced a semiLagrangian particle method (SLPM). In either concept, for FVPM or SLPM, the resulting particle method relies on local scattered data approximation, cf. [13] for further details.

To explain basic features of the FVPM, let $\Xi=\left\{\xi_{1}, \ldots, \xi_{n}\right\} \subset \Omega$ denote a finite point set of particles (i.e., particle positions). Moreover, for any particle $\xi \in \Xi$ we denote its influence area by $V_{\Xi}(\xi) \subset \Omega$. To make a rather straight forward example, the particles' influence areas may, for instance, be given by the Voronoi tiles

$$
V_{\Xi}(\xi)=\left\{x \in \Omega:\|x-\xi\|=\min _{v \in \Xi}\|x-v\|\right\} \subset \Omega \quad \text { for } \xi \in \Xi
$$

of the Voronoi diagram $\mathscr{V}_{\Xi}=\left\{V_{\Xi}(\xi)\right\}_{\xi \in \Xi}$ for $\Xi$, in which case $\mathscr{V}_{\Xi}$ yields a decomposition of $\Omega$ into convex and closed subdomains $V_{\Xi}(\xi) \subset \Omega$ with pairwise disjoint interior, see Figure 1 for illustration.

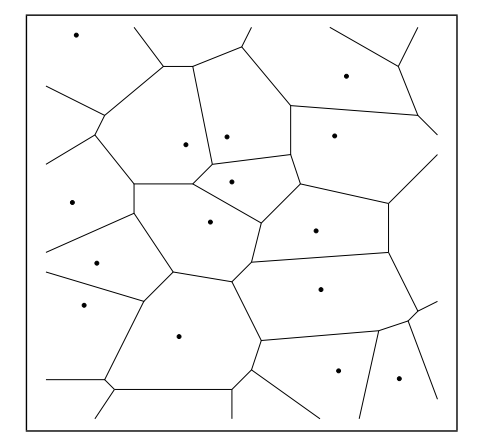

Fig. 1 Finite volume particle method (FVPM). A finite set $\Xi$ of scattered particles $\xi$ (displayed $\bullet$ ) and their influence areas, here given by their Voronoi tiles $V_{\Xi}(\xi)$, are shown. 
Note that the Voronoi diagram $\mathscr{V} \Xi$ is entirely determined by the geometry of the particle distribution in $\Xi$. We remark that there are efficient algorithms from computational geometry [19] for the construction and maintenance of the Voronoi diagram $\mathscr{V}_{\Xi}$ and its dual Delaunay tesselation. The combination between Voronoi diagrams and finite volumes yields through the basic concept of the FVPM a flexible particle method for the numerical solution of (1),(2). We further remark that a more general concept of the FVPM [10,14], allows for overlapping influence areas $\left\{V_{\Xi}(\xi)\right\}_{\xi \in \Xi}$ in which case, however, the FVPM needs to be combined with a partition of unity method (PUM). This provides more flexibility, but it leads to a more complicated FVPM discretization. For more details, we refer to [14].

Now, for any particle located at $\xi \in \Xi$ at time $t$, its particle average is defined by

$$
\bar{u}_{\xi}(t)=\frac{1}{\left|V_{\Xi}(\xi)\right|} \int_{V_{\Xi}(\xi)} u(t, x) d x \quad \text { for } \xi \in \Xi \text { and } t \in[0, T] .
$$

According to the classical concept of finite volume methods [16], for each $\xi \in \Xi$ the average value $\bar{u}_{\xi}(t)$ is, at time step $t \rightarrow t+\tau$, updated by an explicit numerical method of the form

$$
\bar{u}_{\xi}(t+\tau)=\bar{u}_{\xi}(t)-\frac{\tau}{\left|V_{\Xi}(\xi)\right|} \sum_{v} F_{\xi, v}
$$

where $F_{\xi, v}$ denotes the numerical flux between particle $\xi$ and a neighbouring particle $v \in \Xi \backslash \xi$. The required exchange of information between neighbouring particles is modelled via a generic numerical flux function, which may be implemented by using any suitable FV flux evaluation scheme, e.g. by the generalized Godunov approach of high order ADER flux evaluation [22, 23]. For the sake of brevity, we prefer to omit further details concerning flux evaluation.

The following algorithm reflects one basic time step of the FVPM.

\section{Algorithm 1 Finite Volume Particle Method (FVPM).}

INPUT: Time step $\tau>0$, particles $\Xi$, particle averages $\left\{\bar{u}_{\xi}(t)\right\}_{\xi \in \Xi}$ at time $t$.

FOR each $\xi \in \Xi \mathbf{D O}$

(a) Determine set $N_{\xi} \subset \Xi \backslash \xi$ of neighbouring particles around $\xi$;

(b) Compute numerical flux $F_{\xi, v}$ for each $v \in N_{\xi}$;

(c) Update particle average $\bar{u}_{\xi}$ for $\xi$ by (3).

OUTPUT: Particle averages $\left\{\bar{u}_{\xi}(t+\tau)\right\}_{\xi \in \Xi}$ at time $t+\tau$.

\section{WENO Reconstruction}

Modern approaches of FV discretizations are usually combined with essentially non-oscillatory (ENO) [9], or weighted essentially non-oscillatory (WENO) [17] 
reconstruction schemes to obtain conservative, high order numerical methods for hyperbolic conservation laws (1).

To explain how FVPM can be combined with ENO and WENO reconstruction, let us view the influence area $V_{\Xi}(\xi)$ of any particle $\xi \in \Xi$ as the control volume of $\xi$, where the control volume $V_{\Xi}(\xi)$ is uniquely represented by $\xi$.

The basic idea of the ENO method is to first select, for each particle $\xi \in \Xi$, a small set $\left\{S_{i}\right\}_{i=1}^{k}$ of $k$ stencils, where any stencil $S_{i} \subset \Xi$ is given by a set of particles lying in the neighbourhood of $\xi$. Then, for each stencil $S_{i}, 1 \leq i \leq k$, a reconstruction $s_{i} \equiv s_{S_{i}}$ is computed, which interpolates the given particle averages $\left\{\bar{u}_{v}(t): v \in S_{i}\right\}$ over the control volumes $\left\{V_{\Xi}(v)\right\}_{v \in S_{i}}$ of the stencil $S_{i}$.

Among the $k$ different reconstructions $s_{i}, 1 \leq i \leq k$, for the $k$ different stencils $S_{i}$, the smoothest (i.e., the least oscillatory) reconstruction is selected, which constitutes the numerical solution over the control volume $V_{\Xi}(\xi)$. The selection of the smoothest $s_{i}$ among the $k$ reconstructions is done by using a suitable oscillation indicator $\mathscr{I}$.

In a WENO reconstruction, all reconstructions $s_{i}, 1 \leq i \leq k$, are used to construct, for a corresponding control volume $V_{\Xi}(\xi)$, a weighted sum of the form

$$
s(x)=\sum_{i=1}^{k} \omega_{i} s_{i}(x) \quad \text { with } \sum_{i=1}^{k} \omega_{i}=1
$$

where the weights $\omega_{i}=\tilde{\omega}_{i} / \sum_{j=1}^{k} \tilde{\omega}_{j}$, with $\tilde{\omega}_{i}=\left(\varepsilon+\mathscr{I}\left(s_{i}\right)\right)^{-\rho}$ for some $\varepsilon, \rho>0$, are determined by using the aforementioned oscillation indicator $\mathscr{I}$.

We remark that commonly used ENO/WENO schemes work with polynomial reconstruction, which, however, may lead to severe numerical instabilities and other disadvantages, especially for anisotropic distributions of particles, see [1, 2]. In Section 6, we propose a numerically stable reconstruction method of arbitrary high order, which essentially avoids (plain) polynomial reconstruction. The utilized reconstruction relies on a variational formulation, which also provides a natural choice for an efficient oscillation indicator $\mathscr{I}$, as proposed in (8).

\section{Kernel-based Reconstruction in Particle Flow Simulations}

In a generic formulation of particle methods [13], we are essentially concerned with the reconstruction of a numerical solution $u \equiv u(t, \cdot)$, for fixed time $t \in[0, T]$, from its discrete values

$$
u_{\Xi}=\left(u\left(\xi_{1}\right), \ldots, u\left(\xi_{n}\right)\right)^{T} \in \mathbb{R}^{n},
$$

taken at a scattered set $\Xi=\left\{\xi_{1}, \ldots, \xi_{n}\right\} \subset \mathbb{R}^{d}$ of particles, cf. Figure 2 for illustration. In the Eulerian FVPM of the previous section, for instance, the discrete values in $u_{\Xi}$ may be regarded as particle averages of $u$ attached to the particles' positions in $\Xi$. In a semi-Lagrangian particle method (SLPM), the values $u_{\Xi}$ may reflect the concentration of the solution $u$ at upstream locations on the characteris- 
tic curves (streamlines) of the backward flow. For more details concerning relevant Lagrangian and Eulerian particle methods, which we have in mind, we refer to our previous work [13], where suitable prototypes for a finite volume particle method (FVPM) and a semi-Lagrangian particle method (SLPM) are developed.

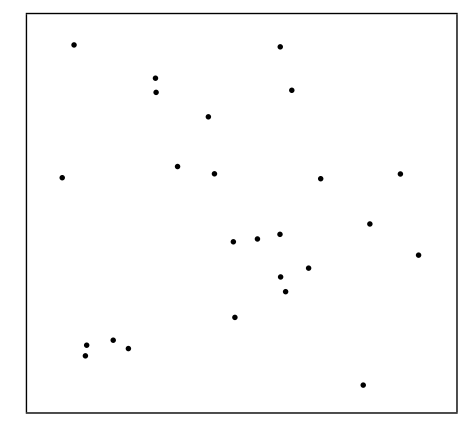

Fig. 2 A finite scattered set $\Xi=\left\{\xi_{1}, \ldots, \xi_{n}\right\} \subset \mathbb{R}^{2}$ of particles. Each particle $\xi$ (displayed $\bullet$ ) bears a scalar function value $u(\xi) \equiv u(t, \xi)$ of the numerical solution $u$ at time $t \in[0, T]$.

Numerical particle flow simulations usually require flexible reconstruction methods from multivariate scattered data approximation to establish, at any time $t$, the coupling between the discrete model for the numerical solution $u$ and its continuous output in the recovery step. To this end, (conditionally) positive definite kernel functions are popular tools.

To explain the basic features of such kernel-based reconstructions, we restrict ourselves to the special case of interpolation, where we seek to compute a suitable interpolant $s: \Omega \rightarrow \mathbb{R}$ satisfying $u_{\Xi}=s_{\Xi}$, i.e.,

$$
u\left(\xi_{k}\right)=s\left(\xi_{k}\right) \quad \text { for all } k=1, \ldots, n .
$$

According to the general formulation of kernel-based interpolation, we assume that the reconstruction $s$ has the form

$$
s(x)=\sum_{j=1}^{n} c_{j} \varphi\left(x-\xi_{j}\right)+p(x) \quad \text { for } p \in \mathscr{P}_{m}^{d},
$$

for some coefficients $c_{1}, \ldots, c_{n} \in \mathbb{R}$, where $\varphi: \Omega \rightarrow \mathbb{R}$ is a fixed (conditionally positive definite) kernel function and $\mathscr{P}_{m}^{d}$ is the linear space of all $d$-variate polynomials of a specific order $m \in \mathbb{N}_{0}$. The required order $m$ in (5) is determined by the choice of $\varphi$. If $m=0$, then the polynomial part in (5) is empty, in which case the reconstruction $s$ has the form

$$
s(x)=\sum_{j=1}^{n} c_{j} \varphi\left(x-\xi_{j}\right)
$$


Rather than dwelling much on explaining conditionally positive definite kernel functions and the structure of their native reproducing kernel Hilbert spaces, we refer to the text books $[5,7,12,24]$. For the following of our discussion, it is sufficient to say that scattered data interpolation by positive definite kernels (where $m=0$ ) leads to a unique reconstruction of the form (6). Moreover, for conditionally positive definite kernels of order $m \in \mathbb{N}$, we obtain under vanishing moment conditions

$$
\sum_{j=1}^{n} c_{j} p\left(\xi_{j}\right)=0 \quad \text { for all } p \in \mathscr{P}_{m}^{d}
$$

a reconstruction $s$ of the form (5), where $s$ is unique, if any polynomial $p \in \mathscr{P}_{m}^{d}$ can uniquely be reconstructed from its values at the points $\Xi$, i.e., $p_{\Xi}=0$ implies $p \equiv 0$.

Let us make examples of commonly used radial kernel functions $\varphi(x)=\phi(\|x\|)$, along with their orders $m \equiv m(\phi)$, where $r=\|x\| \in[0, \infty)$ is, for $x \in \mathbb{R}^{d}$, the radial variable w.r.t. the Euclidean norm $\|\cdot\|$ on $\mathbb{R}^{d}$.

Example 1. The positive definite Gaussian function

$$
\phi(r)=e^{-r^{2}} \quad \text { for } r \in[0, \infty)
$$

is a radial kernel of order $m=0$, so that the reconstruction $s$ has the form (6).

Example 2. The multiquadric

$$
\phi(r)=\left(1+r^{2}\right)^{\beta} \quad \text { for } \beta>0 \text { and } \beta \notin \mathbb{N}
$$

is a conditionally positive definite kernel of order $m=\lceil\beta\rceil$.

The inverse multiquadric

$$
\phi(r)=\left(1+r^{2}\right)^{\beta} \quad \text { for } \beta<0
$$

is positive definite, and so $m=0$. In this case, the reconstruction $s$ has the form (6).

Example 3. The radial characteristic functions [3]

$$
\phi(r)=(1-r)_{+}^{\beta}=\left\{\begin{array}{cc}
(1-r)^{\beta} & \text { for } r<1 \\
0 & \text { for } r \geq 1
\end{array}\right.
$$

are for $d \geq 2$ positive definite on $\mathbb{R}^{d}$, provided that $\beta \geq(d+1) / 2$. In this case, $m=0$, and so the reconstruction $s$ has the form (6).

In the following section, we add polyharmonic splines to the list of our examples. Polyharmonic splines are extraordinarily useful radial kernels, which deserve to be treated in a separate section. Later in this work, we give a strong recommendation in favour of polyharmonic splines, where our supporting arguments will be based on their superior numerical stability at arbitrary high local approximation order. 


\section{Reconstruction by Polyharmonic Splines}

Polyharmonic splines, due to Duchon [6], are traditional tools for Lagrange interpolation from multivariate scattered data. According to the polyharmonic spline interpolation scheme, the reconstruction $s$ has the form (5), where the radial polyharmonic spline kernel $\varphi(x)=\phi_{d, m}(r)$, for $r=\|x\|$, is given as

$$
\phi_{d, m}(r)=\left\{\begin{array}{ll}
r^{2 m-d} \log (r) & \text { for } d \text { even } \\
r^{2 m-d} & \text { for } d \text { odd }
\end{array}\right\} \quad \text { for } 2 m>d
$$

with $m$ being the order of the kernel $\phi_{d, m}$, i.e., $m$ is the order of the polynomial in (5).

According to [6], scattered data interpolation by polyharmonic splines is optimal in its native reproducing kernel Hilbert space, as given by the Beppo Levi space

$$
\mathrm{BL}^{m}\left(\mathbb{R}^{d}\right)=\left\{u: D^{\alpha} u \in L^{2}\left(\mathbb{R}^{d}\right) \text { for all }|\alpha|=m\right\} \subset \mathscr{C}\left(\mathbb{R}^{d}\right),
$$

being equipped with the semi-norm

$$
|u|_{\mathrm{BL}^{m}}^{2}=\sum_{|\alpha|=m}\left(\begin{array}{c}
m \\
\alpha
\end{array}\right)\left\|D^{\alpha} u\right\|_{L^{2}\left(\mathbb{R}^{d}\right)}^{2} .
$$

In other words, the reconstruction $s$ in (5) minimizes the Beppo Levi energy functional $|\cdot|_{\mathrm{BL}^{m}}$ among all recovery functions $u$ in $\mathrm{BL}^{m}\left(\mathbb{R}^{d}\right)$, i.e.,

$$
|s|_{\mathrm{BL}^{m}} \leq|u|_{\mathrm{BL}^{m}}, \quad \text { for all } u \in \mathrm{BL}^{m}\left(\mathbb{R}^{d}\right) \text { with } u_{\Xi}=s_{\Xi} .
$$

Therefore, the energy functional $|\cdot|_{\mathrm{BL}^{m}}$ is a natural choice for the oscillation indicator $\mathscr{I}$ required in the WENO reconstruction of Section 4 (cf. [2]). Hence, we let

$$
\mathscr{I}(u):=|u|_{\mathrm{BL}^{m}}
$$

for the oscillation indicator of the utilized WENO reconstruction. We remark that the semi-norm $|s|_{\mathrm{BL}^{m}}$ of the polyharmonic spline reconstruction $s$ is readily available by the quadratic form

$$
|s|_{\mathrm{BL}^{m}}^{2}=\sum_{j, k=1}^{n} c_{j} c_{k} \phi_{d, m}\left(\left\|\xi_{k}-\xi_{j}\right\|\right)
$$

whose coefficient vector $c=\left(c_{1}, \ldots, c_{n}\right)^{T} \in \mathbb{R}^{n}$ is determined by the solution of the resulting linear system (9). This allows efficient evaluations of the reconstruction's oscillation indicator $\mathscr{I}(s)$, giving the above choice in (8) yet another advantage.

Let us finally discuss the popular special case of thin plate spline reconstruction. In this case, $d=m=2$, so that the thin plate spline kernel is $\phi_{2,2}(r)=r^{2} \log (r)$. Therefore, the reconstruction in (5) has the form 


$$
s(x)=\sum_{j=1}^{n} c_{j}\left\|x-\xi_{j}\right\|^{2} \log \left(\left\|x-\xi_{j}\right\|\right)+d_{0}+d_{1} x_{1}+d_{2} x_{2} \quad \text { for } x=\left(x_{1}, x_{2}\right),
$$

where

$$
\operatorname{BL}^{2}\left(\mathbb{R}^{2}\right)=\left\{u: D^{\alpha} u \in L^{2}\left(\mathbb{R}^{d}\right) \text { for all }|\alpha|=2\right\} \subset \mathscr{C}\left(\mathbb{R}^{2}\right)
$$

is the Beppo-Levi space of second order over $\mathbb{R}^{2}$, whose semi-norm

$$
|u|_{\mathrm{BL}^{2}}^{2}=\int_{\mathbb{R}^{2}}\left(u_{x_{1} x_{1}}^{2}+2 u_{x_{1} x_{2}}^{2}+u_{x_{2} x_{2}}^{2}\right) d x
$$

reflects the bending energy for a thin plate of infinite extent. Since the resulting reconstruction minimizes the bending energy $|\cdot|_{\mathrm{BL}^{2}}$ among all interpolants in $\mathrm{BL}^{2}\left(\mathbb{R}^{2}\right)$, this motivates the naming thin plate spline.

\section{Numerical Aspects of Polyharmonic Spline Reconstruction}

In this section, we discuss the following numerical aspects of polyharmonic splines.

- conditioning of the reconstruction problem;

- numerical stability of the reconstruction algorithm;

- preconditioning and stable implementation of the reconstruction scheme;

- local approximation order.

\subsection{Spectral Condition Number of Reconstruction Matrix}

The coefficients $c=\left(c_{j}\right)_{1 \leq j \leq n} \in \mathbb{R}^{n}$ and $d=\left(d_{\alpha}\right)_{|\alpha|<m}^{T} \in \mathbb{R}^{q}$ of the reconstruction

$$
s(x)=\sum_{j=1}^{n} c_{j} \phi_{d, m}\left(\left\|x-\xi_{j}\right\|\right)+\sum_{|\alpha|<m} d_{\alpha} x^{\alpha}
$$

are given by the solution of the $(n+q) \times(n+q)$ linear system

$$
\left[\begin{array}{cc}
\Phi & P \\
P^{T} & 0
\end{array}\right] \cdot\left[\begin{array}{l}
c \\
d
\end{array}\right]=\left[\begin{array}{c}
u_{\Xi} \\
0
\end{array}\right],
$$

resulting from the interpolation conditions (4), under constraints (7), where

$$
\Phi=\left(\phi_{d, m}\left(\left\|\xi_{k}-\xi_{j}\right\|\right)\right)_{1 \leq j, k \leq n} \in \mathbb{R}^{n \times n} \quad \text { and } \quad P=\left(\xi_{k}^{\alpha}\right)_{1 \leq k \leq n ;|\alpha|<m} \in \mathbb{R}^{n \times q},
$$

with $q=\left(\begin{array}{c}m-1+d \\ d\end{array}\right)$ being the dimension of the polynomial space $\mathscr{P}_{m}^{d}$. We abbreviate the linear system in (9) as 


$$
A \cdot b=u_{\Xi}^{0} .
$$

Since for local reconstruction problems, the number $n$ of interpolation conditions is usually small and since, moreover, the dimension $q$ is small, the dimension of the resulting system (10) is small. In this case, one may be tempted to solve the linear system (10) by a direct solver. This, however, is a rather naive approach for the implementation of the polyharmonic reconstruction scheme, which may lead to severe stability problems. We can explain this as follows.

If the separation distance

$$
q_{\Xi}=\min _{1 \leq j<k \leq n}\left\|\xi_{j}-\xi_{k}\right\|
$$

of the particles in $\Xi$ is small, then the spectral condition number of the system matrix $A$ in (10) will be large. Indeed, if two particles in $\Xi$ are close, then two columns (rows) of the (symmetric) matrix $A$ are almost identical, in which case the smallest eigenvalue of $A$ is close to zero. This important correlation is due to Narcowich and Ward [18], where they prove that the spectral norm of the matrix $\Phi^{-1}$ in (9) is bounded above by a monotonically decreasing function of the separation distance $q_{\Xi}$. This in turns implies that one should, for the sake of numerical stability, avoid solving the system (10) directly for very small $q_{\Xi}$. For further details on this, see [18] and the more general discussion in [20].

To illustrate the spectral behaviour of the reconstruction matrix $A$ in (10), we consider the following numerical experiment. For an initial choice of seven (randomly chosen) interpolation points $\Xi=\left\{x_{0}, x_{1}, \ldots, x_{6}\right\} \subset \mathbb{R}^{2}$ in the plane, as displayed in Figure 3, we computed the spectral condition number $\kappa_{2}(A)$ of the reconstruction matrix $A$, for five different radial kernel functions: Gaussians, multiquadrics, inverse multiquadrics, thin plate splines, and a compactly supported radial kernel.

Further in this numerical experiment, we apply a uniform scaling to the points in $\Xi$ to obtain a sequence of scaled point sets $h \Xi=\left\{h x_{0}, h x_{1}, \ldots, h x_{6}\right\} \subset \mathbb{R}^{2}$, where we let $h=2^{-i}$ for $i=0,1,2,3,4$. Due to the translation invariance of the reconstruction scheme, we assume $x_{0}=0$, without loss of generality, so that the points in $h \Xi$ are, for decreasing $h>0$, shifted towards the origin $x_{0}$, cf. Figure 3 . The sequence of resulting condition numbers $\kappa_{2}(A)$ of the matrices $A \equiv A_{h \Xi}$ are, for the five different radial kernel functions, displayed in the bi-logarithmic plot of Figure 3.

Note that for all five choices of radial kernels, the spectral condition number $\kappa_{2}(A)$ of $A$ is growing at decreasing scale $h>0$, where $\kappa_{2}(A)$ is most rapidly growing for the three smooth kernels, i.e., for the Gaussians and the (inverse) multiquadrics. In fact, there is an intimate correlation between the growth of $\kappa_{2}(A)$ and the smoothness of the kernel $\phi$. This observation is due to Schaback [21], who has developed a general theory for an uncertainty relation between the numerical stability and the approximation order of kernel-based reconstruction schemes.

Given our numerical observations in this rather unsuspicious test case, we can conclude that particular care needs to be taken, when the solution of the reconstruction problem is computed through the linear system (10) - even in situations, where the number of particles (and so the number of interpolation conditions) is small. In 

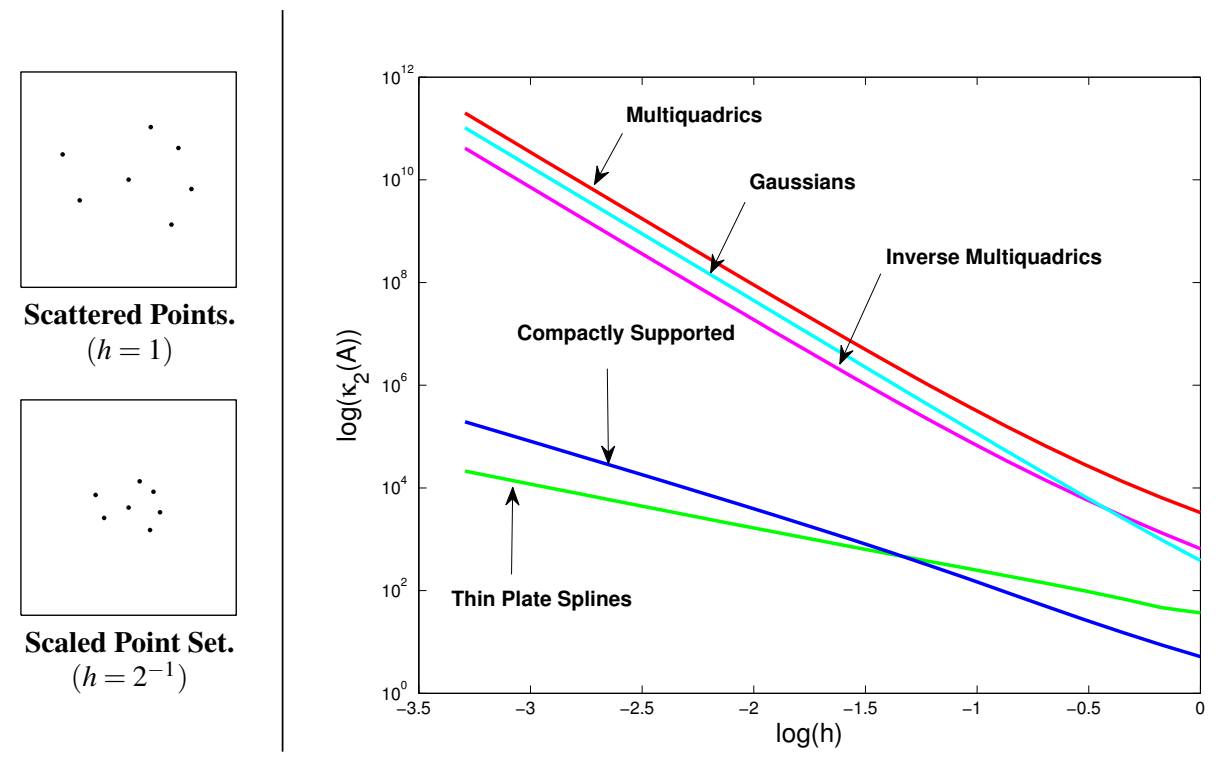

Fig. 3 Spectral condition number $\kappa_{2}(A)$ of reconstruction matrix $A$ for five different radial kernels.

fact, as soon as two particles are close, this may spoil the numerical stability of the reconstruction scheme, when a direct method for the numerical solution of the system (10) is applied. In such critical situations, one should rather consider applying preconditioning techniques. In the following of this paper, we will develop a suitable preconditioner for the polyharmonic spline reconstruction method. But before doing so, let us first analyze the conditioning of the reconstruction problem.

\subsection{Conditioning of Reconstruction Problem}

Recall that the absolute condition number of the reconstruction problem is given by the smallest number $\kappa_{\infty} \equiv \kappa_{\infty, \Xi}$ satisfying

$$
\left\|I_{\Xi} u\right\|_{L_{\infty}(\Omega)} \leq \kappa_{\infty} \cdot\|u\|_{L_{\infty}(\Omega)} \quad \text { for all } u \in \mathscr{C}(\Omega),
$$

where $I_{\Xi}$ is the interpolation operator, which maps, on given $\Xi$, any $u \in \mathscr{C}(\Omega)$ onto its unique polyharmonic spline reconstruction $s$ satisfying $u_{\Xi}=s_{\Xi}$. Therefore, $\kappa_{\infty}$ is the operator norm $\left\|I_{\Xi}\right\|_{\infty}$ of $I_{X}$ w.r.t. the norm $\|\cdot\|_{L_{\infty}(\Omega)}$ on $\mathscr{C}(\Omega)$, i.e., $\kappa_{\infty}=\left\|I_{\Xi}\right\|_{\infty}$.

Now, the operator norm $\left\|I_{\Xi}\right\|_{\infty}$, and so the absolute condition number $\kappa_{\infty}$, can be computed as follows.

Theorem 1. For $\Xi=\left\{\xi_{1}, \ldots, \xi_{n}\right\} \subset \Omega$, the norm $\left\|I_{\Xi}\right\|_{\infty}$ of the interpolation operator $I_{\Xi}$ is given by the Lebesgue constant 


$$
\Lambda_{\infty}:=\max _{x \in \Omega} \sum_{j=1}^{n}\left|\lambda_{j}(x)\right|=\max _{x \in \Omega}\|\lambda(x)\|_{1}
$$

where $\left\{\lambda_{j}\right\}_{j=1}^{n}$ are the Lagrange basis functions of the reconstruction, satisfying

$$
\lambda_{j}\left(\xi_{k}\right)=\delta_{j k}=\left\{\begin{array}{l}
1 \text { for } j=k \\
0 \text { for } j \neq k
\end{array}\right.
$$

For the convenience of the reader, we provide the proof of this general result.

Proof. For any $u \in \mathscr{C}(\Omega)$, let $s=I_{\Xi}(u)$ denote the unique interpolant to $u$ on $\Xi$ satisfying $u_{\Xi}=s_{\Xi}$. Using the (unique) Lagrange representation

$$
s(x)=\sum_{j=1}^{n} \lambda_{j}(x) u\left(\xi_{j}\right)
$$

of the reconstruction $s$, we obtain the estimate

$$
\left\|I_{\Xi}(u)\right\|_{L_{\infty}(\Omega)}=\|s\|_{L_{\infty}(\Omega)} \leq \max _{x \in \Omega} \sum_{j=1}^{n}\left|\lambda_{j}(x)\right| \cdot\left|u\left(x_{j}\right)\right| \leq \Lambda_{\infty} \cdot\|u\|_{L_{\infty}(\Omega)},
$$

and therefore $\left\|I_{\Xi}\right\|_{\infty} \leq \Lambda_{\infty}$.

In order to see that $\left\|I_{\Xi}\right\|_{\infty} \geq \Lambda_{\infty}$, suppose that the maximum of $\Lambda_{\infty}$ in (11) is attained at $x^{*} \in \Omega$. Moreover, let $g \in \mathscr{C}(\Omega)$ be satisfying $g\left(\xi_{j}\right)=\operatorname{sign}\left(\lambda_{j}\left(x^{*}\right)\right)$, for all $1 \leq j \leq n$, and $\|g\|_{L_{\infty}(\Omega)}=1$. Then,

$$
\left\|I_{\Xi}(g)\right\|_{L_{\infty}(\Omega)} \geq\left(I_{\Xi}(g)\right)\left(x^{*}\right)=\sum_{j=1}^{n} \lambda_{j}\left(x^{*}\right) g\left(\xi_{j}\right)=\sum_{j=1}^{n}\left|\lambda_{j}\left(x^{*}\right)\right|=\Lambda_{\infty}
$$

and so $\left\|I_{\Xi}(g)\right\|_{L_{\infty}(\Omega)} \geq \Lambda_{\infty}$, which implies $\left\|I_{\Xi}\right\|_{\infty} \geq \Lambda_{\infty}$. Altogether, $\left\|I_{\Xi}\right\|_{\infty}=\Lambda_{\infty}$.

\subsection{Scale-Invariance of the Lebesgue Constant}

Now let us return to our numerical experiment of Subsection 7.1, where in this section, we record the Lebesgue constants $\Lambda_{\infty} \equiv \Lambda_{\infty}(\Omega, \Xi)$ for the same sequence of scaled point sets $h \Xi$, and for the five different radial kernels as in Subsection 7.1. The resulting behaviour of the Lebesgue constants $\Lambda_{\infty}$ is shown in Figure 4. Note that the Lebesgue constant $\Lambda_{\infty}$ of the thin plate spline reconstruction scheme is scale-invariant. In contrast, for any of the other four kernel functions, the Lebesgue constant, and so the conditioning of the reconstruction problem, is (strictly) monotonically increasing at decreasing scaling $h>0$.

We can prove the scale invariance for the general case of polyharmonic splines, where our arguments rely on the scale invariance of their Lagrange basis functions in combination with the representation of the Lebesgue constant in (11). 

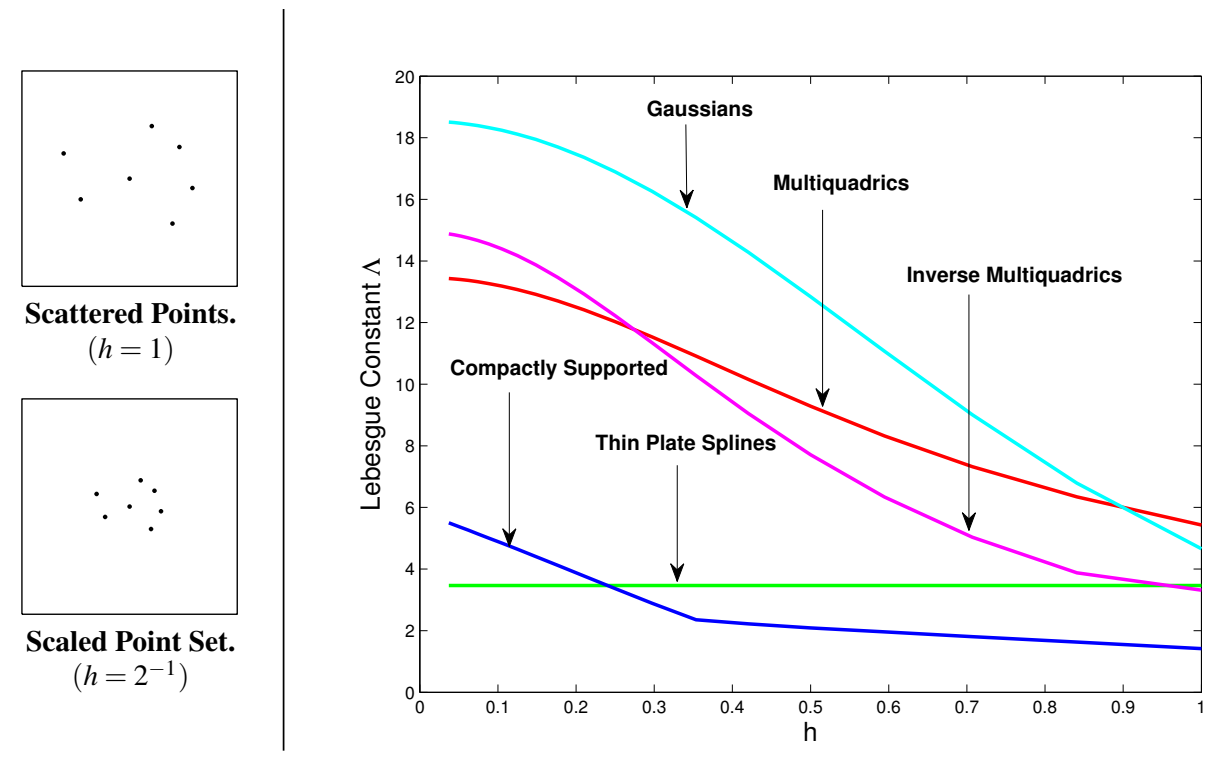

Fig. 4 Lebesgue constant $\Lambda_{\infty} \equiv \Lambda(\Omega, h \Xi)$ for five different radial kernels, where $h=2^{-i}$.

Theorem 2. The Lagrange basis functions $\left\{\lambda_{j}\right\}_{j=1}^{n}$ are scale-invariant.

Proof (sketch): Following our work [11], we see that the reconstruction space

$$
\mathscr{R}=\left\{s=\sum_{j=1}^{n} c_{j} \phi\left(\left\|\cdot-\xi_{j}\right\|\right): \sum_{j=1}^{n} c_{j} p\left(\xi_{j}\right)=0 \text { for all } p \in \mathscr{P}_{m}^{d}\right\}
$$

containing all possible polyharmonic spline reconstructions of the form (5) is invariant under uniform scalings, i.e., for any $h>0$ we find $\mathscr{R}^{h}=\mathscr{R}$, where

$$
\mathscr{R}^{h}=\left\{\sigma_{h}(s): s \in \mathscr{R}\right\}
$$

denotes the scaled reconstruction space, and where $\sigma_{h}$ is the dilation operator, being given by $\sigma_{h}(s)=s(\cdot / h)$. Given uniqueness of the Lagrange functions in either space, $\mathscr{R}$ or $\mathscr{R}^{h}$, this implies

$$
\sigma_{h}\left(\lambda_{j}(x)\right)=\lambda_{j}(x / h)=\lambda_{j}^{h}(x),
$$

where $\left\{\lambda_{j}^{h}\right\}_{j=1}^{n}$ denotes the Lagrange basis in $\mathscr{R}^{h}$.

The above theorem immediately implies that the Lebesgue constant $\Lambda_{\infty}$ in (11) is scale-invariant. Since the polyharmonic spline reconstruction scheme is also invariant under translations and rotations, this yields the following important result.

Corollary 1. The Lebesgue constant $\Lambda_{\infty}$ of polyharmonic spline reconstruction is invariant under translations, rotations, and uniform scalings. 


\subsection{Stable Evaluation of the Reconstruction}

Now the scale invariance of the polyharmonic spline reconstructions' absolute condition number allows us to construct a simple preconditioner to obtain a stable evaluation of the reconstruction $s$ in (5). To this end, we regard for any $h>0$ the scaled reconstruction problem $s_{h \Xi}^{h}=u_{h \Xi}$, i.e.,

$$
s^{h}\left(h \xi_{j}\right)=u\left(h \xi_{j}\right) \quad \text { for all } j=1, \ldots, n,
$$

where $s^{h}$ denotes the unique polyharmonic spline interpolant to the given input data $u_{h \Xi}$. The coefficients of the reconstruction $s^{h}$ can be computed by the scaled linear system

$$
\left[\begin{array}{cc}
\Phi_{h} & P_{h} \\
P_{h}^{T} & 0
\end{array}\right] \cdot\left[\begin{array}{l}
c^{h} \\
d^{h}
\end{array}\right]=\left[\begin{array}{c}
u_{h \Xi} \\
0
\end{array}\right]
$$

which we abbreviate as

$$
A_{h} \cdot b^{h}=u_{h \Xi}^{0} .
$$

Moreover, for any $x \in \mathbb{R}^{d}$, the Lagrange basis functions

$$
\lambda^{h}(h x)=\left(\lambda_{1}^{h}(h x), \ldots, \lambda_{n}^{h}(h x)\right)^{T} \in \mathbb{R}^{n}
$$

are given by the solution of the linear system

$$
\left[\begin{array}{cc}
\Phi_{h} & P_{h} \\
P_{h}^{T} & 0
\end{array}\right] \cdot\left[\begin{array}{c}
\lambda^{h}(h x) \\
\mu^{h}(h x)
\end{array}\right]=\left[\begin{array}{c}
\phi_{d, k}\left(\left\|h\left(x-\xi_{j}\right)\right\|\right)_{j} \\
\left((h x)^{\alpha}\right)_{\alpha}
\end{array}\right],
$$

which we abbreviate as

$$
A_{h} \cdot \kappa^{h}(h x)=\beta_{h}(h x) .
$$

Indeed, if we let $x=\xi_{k}$, for any $1 \leq k \leq n$, then the right hand side $\beta_{h}\left(h \xi_{k}\right)$ of the linear system coincides with the $k$-th column of the matrix $A_{h}$, and so $\lambda^{h}\left(h \xi_{k}\right)$ is the $k$-th unit vector in $\mathbb{R}^{n}$, i.e., we have $\lambda_{j}^{h}\left(h \xi_{k}\right)=\delta_{j k}$.

Now, a stable evaluation of the reconstruction $s^{h}$ at $h x$ relies on its Lagrange representation

$$
s^{h}(h x)=\sum_{j=1}^{n} \lambda_{j}^{h}(h x) u\left(h \xi_{j}\right),
$$

which we can further rewrite as

$$
\begin{aligned}
s^{h}(h x) & =\left\langle\lambda^{h}(h x), u_{h \Xi}\right\rangle=\left\langle\lambda^{1}(x), u_{h \Xi}\right\rangle=\left\langle\kappa^{1}(x), u_{h \Xi}^{0}\right\rangle \\
& =\left\langle A_{1}^{-1} \cdot \beta_{1}(x), u_{h \Xi}^{0}\right\rangle=\left\langle\beta_{1}(x), A_{1}^{-1} \cdot u_{h \Xi}^{0}\right\rangle,
\end{aligned}
$$

where $\langle\cdot, \cdot\rangle$ denotes the usual Euclidean inner product.

In conclusion, by using the above representation for $s^{h}$, the evaluation of the polyharmonic spline reconstruction $s^{h}$ at $h x$ can be accomplished by solving the linear system 


$$
A_{1} b=u_{h \Xi}^{0}
$$

for $b \in \mathbb{R}^{n+q}$, where the spectral condition number $\kappa_{2}\left(A_{1}\right)$ of matrix $A_{1}$ is, for small $0<h \ll 1$, much smaller than the spectral condition number $\kappa_{2}\left(A_{h}\right)$ of $A_{h}$. In this way, we can avoid the direct solution of the ill-conditioned system in (12), by solving the well-conditioned system in (14). This can be viewed as a simple way of preconditioning the scaled system (12), giving a stable method for the evaluation of $s^{h}$, provided that the absolute condition number of the given reconstruction problem not too large.

\subsection{Local Approximation Order}

Now let us turn to the approximation properties of the polyharmonic spline reconstruction method. Recall that we are only interested in local scattered data interpolation, which motivates the following definition.

Definition 1. For $h>0$, let $s^{h}$ be the polyharmonic spline reconstruction satisfying

$$
u\left(h \xi_{j}\right)=s^{h}\left(h \xi_{j}\right), \quad \text { for all } 1 \leq j \leq n .
$$

Then, $p$ is said to be the local approximation order of the polyharmonic spline reconstruction scheme, iff

$$
\left|u(h x)-s^{h}(h x)\right|=\mathscr{O}\left(h^{p}\right), \quad \text { for } h \rightarrow 0, \quad \text { for all } u \in \mathscr{C}^{p} .
$$

Due to our earlier paper [8], the local approximation order of thin plate splines, using the kernel $\phi_{2,2}$, is $p=2$. We have generalized this result in [11] to polyharmonic splines to obtain arbitrary high local approximation orders.

Theorem 3. The local approximation order of polyharmonic spline reconstruction w.r.t. $\mathscr{C}^{m}$, using $\phi_{d, m}$, is $m$.

We can sketch the proof of this important result as follows.

Proof (sketch). Regard for $h>0$ and $x \in \mathbb{R}^{d}$ the $m$-th order Taylor polynomial

$$
T_{u, h x}^{m}(y)=\sum_{|\alpha|<m} \frac{1}{\alpha !} D^{\alpha} u(h x)(y-h x)^{\alpha}
$$

of $u \in \mathscr{C}^{m}$ around $h x$. This yields the identity

$$
u(h x)=T_{u, h x}^{m}\left(h \xi_{j}\right)-\sum_{0<|\alpha|<m} \frac{1}{\alpha !} D^{\alpha} u(h x)\left(h \xi_{j}-h x\right)^{\alpha} \quad \text { for all } j=1, \ldots, n .
$$

By using the Lagrange representation of the reconstruction $s^{h}$ in (13) in combination with the polynomial reproduction property 


$$
p(h x)=\sum_{j=1}^{n} \lambda^{h}(h x) p\left(h \xi_{j}\right) \quad \text { for all } p \in \mathscr{P}_{m}^{d}
$$

this immediately implies the representation

$$
u(h x)-s^{h}(h x)=\sum_{j=1}^{n} \lambda_{j}^{h}(h x)\left[T_{u, h x}^{m}\left(h \xi_{j}\right)-u\left(h \xi_{j}\right)\right]
$$

for the pointwise error at $h x$. Now, due to the scale-invariance of the Lagrange basis, the Lebesgue function

$$
\Lambda(x):=\sum_{j=1}^{n}\left|\lambda_{j}^{h}(h x)\right|=\sum_{j=1}^{n}\left|\lambda_{j}^{1}(x)\right|
$$

is uniformly bounded in any local neighbourhood of the origin. Since we have

$$
T_{u, h x}^{m}\left(h \xi_{j}\right)-u\left(h \xi_{j}\right)=\mathscr{O}\left(h^{m}\right), \quad \text { for } h \rightarrow 0, \quad \text { for all } 1 \leq j \leq n,
$$

this then implies

$$
\left|u(h x)-s^{h}(h x)\right|=\mathscr{O}\left(h^{m}\right) \quad \text { for } h \rightarrow 0 .
$$

\subsection{Advantages of Polyharmonic Spline Reconstruction}

Let us draw an intermediate conclusion on polyharmonic spline reconstruction.

Well-posedness. We have shown that polyharmonic spline interpolation leads to a well-posed reconstruction method, which works for arbitrary distributions of scattered interpolation points $\Xi \subset \mathbb{R}^{d}$, for arbitrary function values $u_{\Xi}$, and in arbitrary space dimensions $d \geq 1$.

Optimality. The polyharmonic spline reconstruction scheme is optimal in its corresponding Beppo-Levi space $\mathrm{BL}^{m}\left(\mathbb{R}^{d}\right)$, being the native reproducing kernel Hilbert space of the polyharmonic spline kernel $\phi_{d, m}$. We remark that the optimal recovery space $\mathrm{BL}^{m}\left(\mathbb{R}^{d}\right)$ is the Sobolev space $\mathscr{H}^{m}\left(\mathbb{R}^{d}\right)$, which is a relevant function space in nonlinear hyperbolic problems.

Meshfree reconstruction and high flexibility. The reconstruction scheme of polyharmonic splines is meshfree and therefore very flexible, especially when it comes to adaptively modifying the set of moving particles. This property is particularly important for problems with solutions of rapid variation or singularities, or for problems with free or complicated boundaries as well as for various other challenging problems, where the simulation of multiscale phenomena is an issue.

Efficient implementation. The implementation of the polyharmonic spline reconstruction scheme requires solving a square linear system (9), which is small, if the number of particles in $\Xi$ is small. On the down side, the linear system (9) is ill-conditioned, if the separation distance $q_{\Xi}$ of the particles in $\Xi$ is small. 
Before we further discuss the properties of polyharmonic splines, let us make a comparison with the other four types of kernel functions, from Examples 1-3, where the following three remarks are in order.

Remark 1. The above mentioned advantages of the polyharmonic spline reconstruction scheme are also satisfied for the above mentioned alternative choices of radial kernels, i.e., for the Gaussians, the (inverse) multiquadrics, and for the compactly supported radial kernels, from Examples 1-3.

Remark 2. For the smooth kernels, i.e., for the Gaussians and for the (inverse) multiquadrics, their corresponding optimal recovery spaces are much smaller than the Beppo-Levi space $\mathrm{BL}^{m}\left(\mathbb{R}^{d}\right)$ from the polyharmonic spline kernels $\phi_{d, m}$. In fact, their restrictive native spaces are mainly consisting of band-limited functions, which are (compared with the Sobolev spaces $\mathrm{BL}^{m}\left(\mathbb{R}^{d}\right)$ ) less relevant for hyperbolic problems.

Remark 3. The key property of the polyharmonic spline reconstruction scheme is the scale-invariance of their Lagrange basis functions. This important property is not satisfied for the other four types of kernel functions, i.e., the Lagrange basis functions of the Gaussians, of the (inverse) multiquadrics and of the compactly supported radial kernels, from Examples 1-3, are not scale-invariant.

Note that the last remark implies that the preconditioner for polyharmonic splines, as developed in Subsection 7.4, is not available for the other four types of radial kernel functions. Moreover, our results of Subsection 7.5 concerning the local approximation order do only apply to polyharmonic splines. This gives the polyharmonic spline reconstruction scheme yet another a major advantage over the other four radial kernels. We can summarize this as follows.

Numerical stability. The polyharmonic spline reconstruction scheme allows for numerically stable evaluations of the interpolant, with using the preconditioner of Subsection 7.4.

Arbitrary local approximation order. Polyharmonic spline reconstruction has local approximation order $m$ w.r.t. $\mathscr{C}^{m}$ functions, with using the kernel $\phi_{d, m}$.

We conclude our discussion by giving a strong recommendation in favour of polyharmonic splines, especially when they are used in combination with adaptive particle methods for numerical flow simulation, where highly flexible and sufficiently robust reconstructions of arbitrary order are needed. This recommendation is in particular supported by our numerical experiments in Section 9.

\section{Adaption Rules}

Let us finally discuss the construction and implementation of customized adaption rules, as they are required for dynamic modifications of the moving particles. 


\subsection{Error Indication}

An effective strategy for the adaptive modification of the particle set requires wellmotivated refinement and coarsening rules as well as a customized error indicator. We understand the error indicator $\eta: \Xi \rightarrow[0, \infty)$ as a function of the particle set $\Xi$ which assigns a significance value $\eta(\xi)$ to each particle $\xi \in \Xi$. The significance $\eta(\xi)$ is required to reflect the local approximation quality of the reconstruction around $\xi \in \Xi$.

Our proposed adaption rules rely on the error indicator

$$
\eta(\xi)=\left|u(\xi)-s_{N_{\xi}}(\xi)\right| \quad \text { for } \xi \in \Xi,
$$

where $s_{N_{\xi}}$ is the polyharmonic spline reconstruction which matches the solution's values at a particle set $N_{\xi} \subset \Xi \backslash \xi$ in a neighbourhood around $\xi$, but not at $\xi$, i.e.,

$$
s_{N_{\xi}}(v)=u(v) \quad \text { for all } v \in N_{\xi},
$$

see Figure 5 for illustration.

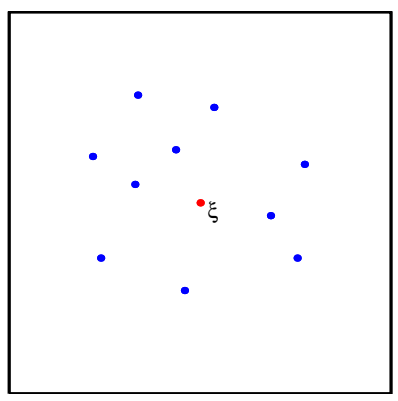

Fig. 5 Error indication for particle $\xi \in \Xi$. The polyharmonic spline reconstruction $s_{N_{\xi}}$ interpolates $u$ at the points $N_{\xi} \subset \Xi \backslash \xi$ in a local neighbourhood around $\xi \in \Xi$, but not at $\xi$. The deviation between the interpolated value $s_{N_{\xi}}(\xi)$ and $u(\xi)$ yields an error indication $\eta(\xi)$ at $\xi$.

Therefore, the error indicator measures the deviation between the known function value $u(\xi)$ of the solution and the interpolated value around the particle $\xi$. When $\xi$ lies in a smooth region of the solution, the error indication $\eta(\xi)$ is expected to be small, whereas in regions of less regularity for $u$, or around discontinuities, the error indication $\eta(\xi)$ is expected to be large.

The significances $\eta(\xi), \xi \in \Xi$, are used in order to flag single particles $\xi \in \Xi$ as "to be refined" or "to be coarsened" according to the following criteria.

Definition 2. Let $\eta^{*}=\max _{\xi \in \Xi} \eta(\xi)$, and let $\theta_{\mathrm{crs}}, \theta_{\text {ref }}$ be two tolerance values satisfying $0<\theta_{\mathrm{crs}}<\theta_{\text {ref }}<1$. We say that a particle $\xi \in \Xi$ is to be refined, iff $\eta(\xi)>\theta_{\text {ref }} \cdot \eta^{*}$, and $\xi$ is to be coarsened, iff $\eta(\xi)<\theta_{\mathrm{crs}} \cdot \eta^{*}$. 
In our numerical examples typical choices for the relative tolerance values are $\theta_{\text {crs }}=0.1$ and $\theta_{\text {ref }}=0.2$. Note that a particle $\xi$ cannot be refined and be coarsened at the same time; in fact, it may neither be refined nor be coarsened.

\subsection{Coarsening and Refinement}

In order to balance the approximation quality of the model against the required computational complexity we insert new particles into regions where the value of the error indicator $\eta$ is high (refinement), whereas we remove particles from $\Xi$ in regions where the value of $\eta$ is small (coarsening).

To avoid additional computational overhead and complicated data structures, effective adaption rules are required to be as simple as possible. In particular, these rules ought to be given by local operations on the current particle set $\Xi$. The following coarsening rule is in fact very simple to implement and, in combination with our refinement rule, it turns out to be very effective as well.

Definition 3. A particle $\xi \in \Xi$ is coarsened by its removal from the current particle set $\Xi$, i.e. $\Xi$ is modified by letting $\Xi=\Xi \backslash \xi$.

As concerns the refinement of a particle $\xi \in \Xi$, our starting point for the design of a customized refinement rule is motivated by available local error estimates for polyharmonic spline interpolation, which are, for a local region $U \subset \Omega$ of the computational domain $\Omega$ relying on the local fill distance on $U$,

$$
h \equiv h_{\Xi}(U)=\sup _{y \in U} d_{\Xi}(y),
$$

where $d_{\Xi}(y)=\min _{\xi \in \Xi}\|y-\xi\|$ is the distance between the $y$ and the point set $\Xi$.

Therefore, $h_{\Xi}(U)$ reflects the local density of points $\Xi$ around $U$ by giving the largest Euclidean distance between a point in $U$ and its nearest particle in $\Xi$. To reduce the norm of the pointwise error functional of the polyharmonic spline reconstruction scheme on $U$, the local fill distance $h_{\Xi}(U)$ should be reduced.

This motivates us to construct the refinement rule as follows. Regarding the local fill distance $h_{\Xi}(U)$ in a local neighbourhood $U \equiv U(\xi)$ of a particle $\xi$, we wish to insert only a few new particles into $U$, such that the resulting local fill distance around $\xi$ is reduced as much as possible.

We remark that the distance function $d_{\Xi}: \Omega \rightarrow[0, \infty)$ is a convex function which attains local maxima in the local neighbourhood $U$ of $\xi$ at the Voronoi vertices of the Voronoi tile $V_{\Xi}(\xi)$, see Figure 6 for illustration.

This gives rise to define the refinement of a particle as follows.

Definition 4. A particle $\xi \in \Xi$ is refined by the insertion of the vertices of the Voronoi tile $V_{\Xi}(\xi)$ into the particle set $\Xi$. 


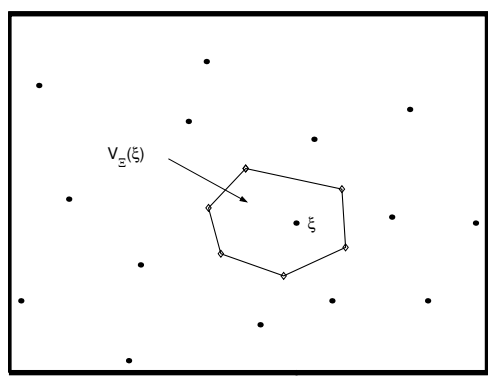

Fig. 6 Refinement of particle $\xi \in \Xi$. The Voronoi vertices $(\diamond)$ of Voronoi tile $V_{\Xi}(\xi)$ are inserted.

\section{Oil Reservoir Simulation: The Five-Spot Problem}

In this section, we recap selected numerical results from our previous work [13] for the purpose of illustration, where all numerical simulations were performed by using the FVPM in combination with WENO reconstruction by polyharmonic splines.

\subsection{The Five-Spot Problem}

The following variant of the five-spot problem in two dimensions, $d=2$, may be summarized as follows. The computational domain $\Omega=[-0.5,0.5]^{2}$ is corresponding to a bounded reservoir, where we assume, for the sake of simplicity, unit permeability of a homogeneous porous medium.

Initially, the pores of the reservoir are saturated with non-wetting fluid (oil), before wetting fluid (water) is injected through one injection well, being placed at the center of $\Omega$. During the simulation, the non-wetting fluid (oil) is displaced by the wetting fluid (water) towards the four corners of the square domain $\Omega$.

The five-spot problem requires solving the following set of three coupled equations: the Buckley-Leverett equation

$$
\frac{\partial u}{\partial t}+\mathbf{v} \cdot \nabla f(u)=0
$$

with fractional flow function

$$
f(u)=\frac{u^{2}}{u^{2}+\mu(1-u)^{2}},
$$

$\mu=\mu_{w} / \mu_{o}$ being the ratio of the two fluids' viscosities, $\mu_{w}$ (water) and $\mu_{o}$ (oil), together with the incompressibility relation

$$
\nabla \cdot \mathbf{v}(t, x)=0
$$


and Darcy's law

$$
\mathbf{v}(t, x)=-M(u) \nabla p(t, x),
$$

describes the flow of two immiscible incompressible fluids, water and oil, through a porous homogeneous medium, in the absence of capillary pressure and gravitational effects.

The solution $u$ of (15),(17),(18) is the saturation of the wetting fluid (water). Hence, the value $u(t, x)$ is, at a time $t$ and at a point $x$, the fraction of available volume (in the pores of the medium) filled with water, and so $u=1$ means pure water, and $u=0$ means pure oil. Figure 7 shows the contour lines of the pressure field $p$ together with the streamlines of the velocity field $\mathbf{v}$, resulting from Darcy's law (18).

We consider solving the above equation system (15),(17),(18) on $\Omega$, in combination with the initial condition

$$
u_{0}(x)= \begin{cases}1 & \text { for }\|x\| \leq R \\ 0 & \text { otherwise }\end{cases}
$$

where we let $R=0.02$ for the radius of the injection well.

\subsection{Adaptive Particle Flow Simulation}

We apply our adaptive particle method to the Cauchy problem (15),(19) for the Buckley-Leverett equation. Recall that this is in order to model the propagation of the shock front, which is of primary importance in the relevant application, where the accurate approximation of the shock front requires particular care. This is in our method mainly accomplished by the adaptive modification of the particles during the simulation. For details concerning the construction of the required adaption rules, we refer to [4].

Now let us turn straight to our numerical results, provided by our particle advection scheme. In our simulation, we decided to select a constant time step size $\tau=5 \cdot 10^{-5}$, and the simulation comprises 2100 time steps, so that $I=[0,2100 \tau]$. Moreover, we let $\mu=0.5$ for the viscosity ratio of water and oil, appearing in the fractional flow function (16).

The initial conditions $u(0, x)$ are shown in Figure 8, where also the initial particle distribution is shown. Moreover, Figure 9 shows the water saturation $u$ during the simulation at three different times, $t=t_{420}, t=t_{1260}$, and $t=t_{2100}$. Figure 9 shows also the corresponding particle distribution. The colour code for the water saturation is shown at the right margin of Figure 9, respectively.

Note that the shock front, at the interface between the non-wetting fluid (oil, $u \equiv 0$ ) and the wetting fluid (water, $u \equiv 1$ ), is moving from the center towards the four corner points of the computational domain $\Omega$. This way, the non-wetting fluid (oil) is effectively displaced by the wetting fluid (water) into the four production wells, as expected. 


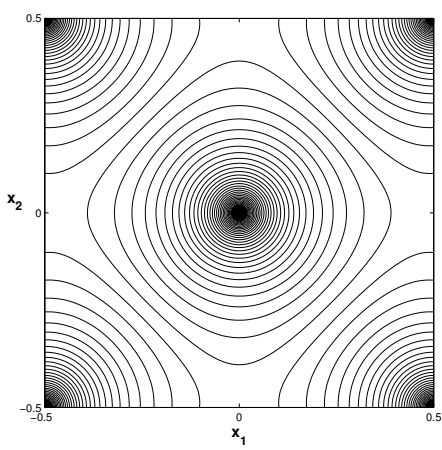

(a)

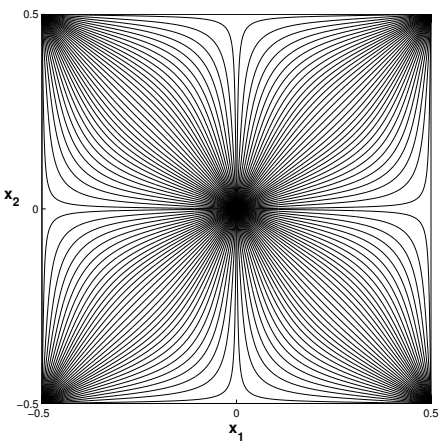

(b)

Fig. 7 Five-spot problem. (a) Contours of the pressure field, (b) streamlines of the velocity field.

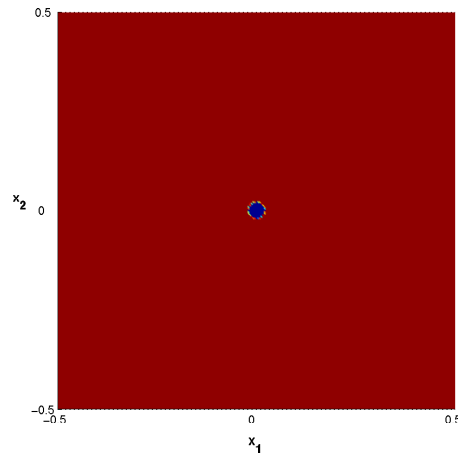

(a) $t=t_{0}$

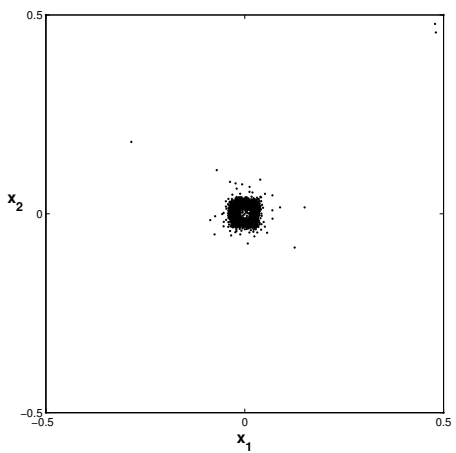

(b) $t=t_{0}$

Fig. 8 Five-spot problem. (a) Initial condition, (b) initial particle distribution.

Due to the adaptive distribution of the particles, the shock front propagation of the solution $u$ is captured very well. This helps to reduce the required computational costs while maintaining the accuracy, due to a higher resolution around the shock front. The effective distribution of the particles around the shock supports the utility of the adaption rules, proposed in our previous paper [4], yet once more.

Acknowledgements This work is partly supported by the priority program DFG-SPP 1324 of the Deutsche Forschungsgemeinschaft (DFG) within the project IS 58/1-2. 

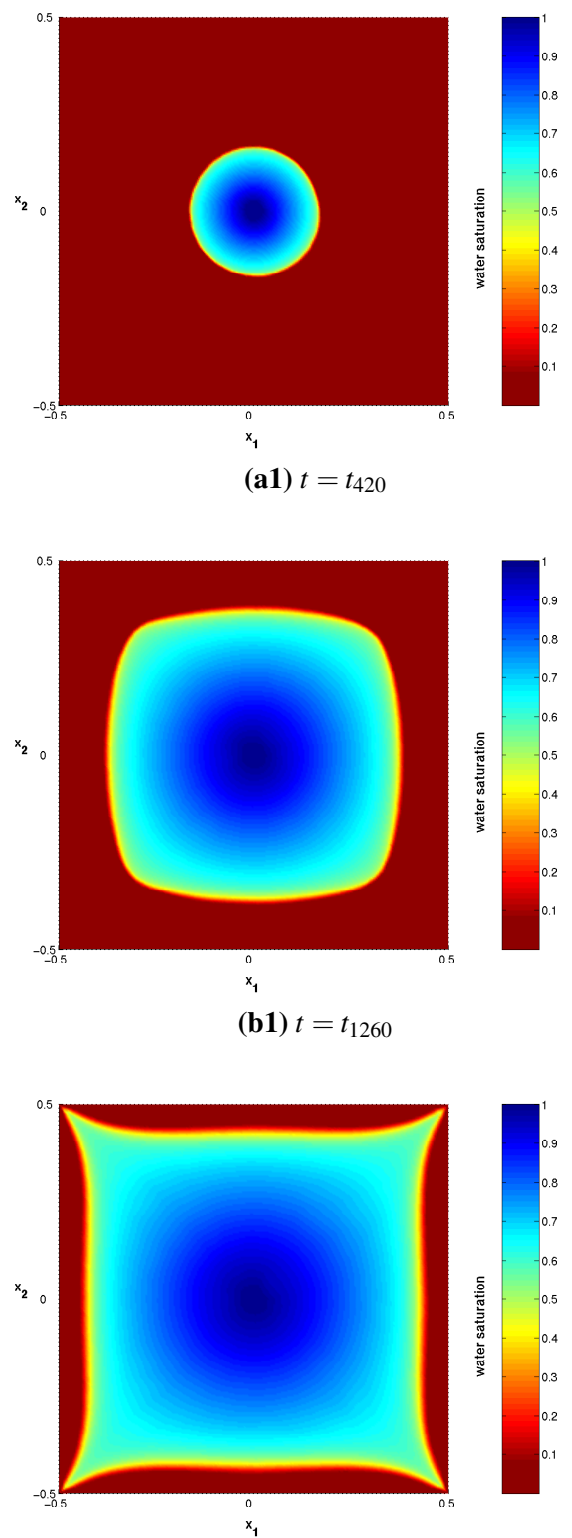

(a1) $t=t_{420}$

(b1) $t=t_{1260}$

(c1) $t=t_{2100}$
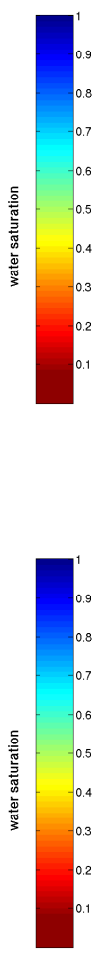

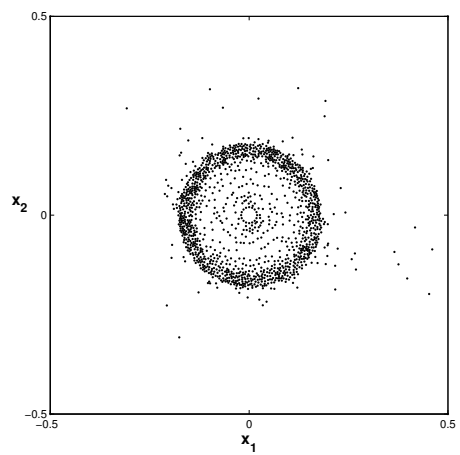

(a2) $t=t_{420}$

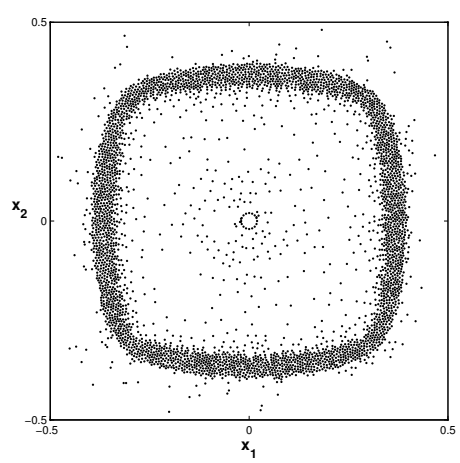

(b2) $t=t_{1260}$

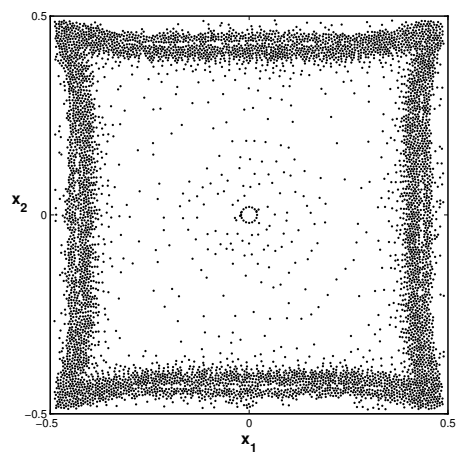

(c2) $t=t_{2100}$

Fig. 9 Five-spot problem. Solution obtained by our particle simulation. The colour plots indicate the water saturation $u$ during the simulation at three different times, (a1) $t=t_{420}$, (b1) $t=t_{1260}$, (c1) $t=t_{2100}$. The corresponding adaptive particle distributions are shown in (a2),(b2),(c2). 


\section{References}

1. R. Abgrall: On essentially non-oscillatory schemes on unstructured meshes: analysis and implementation. J. Comput. Phys. 144, 1994, 45-58.

2. T. Aboiyar, E.H. Georgoulis, and A. Iske: Adaptive ADER methods using kernel-based polyharmonic spline WENO reconstruction. SIAM J. Scient. Computing 32(6), 2010, 3251-3277.

3. R. Askey: Radial Characteristic Functions. Technical report TSR \# 1262, University of Wisconsin, Madison, 1973.

4. J. Behrens, A. Iske, and S. Pöhn: Effective node adaption for grid-free semi-Lagrangian advection. In: Discrete Modelling and Discrete Algorithms in Continuum Mechanics, T. Sonar and I. Thomas (eds.), Logos, 2001, 110-119.

5. M.D. Buhmann: Radial Basis Functions: Theory and Implementations. Cambridge University Press, Cambridge, UK, 2003.

6. J. Duchon: Splines minimizing rotation-invariant semi-norms in Sobolev spaces. In: Constructive Theory of Functions of Several Variables, W. Schempp and K. Zeller (eds.), Springer, 1977, 85-100.

7. G.E. Fasshauer: Meshfree Approximation Methods with Matlab. Interdisciplinary Mathematical Sciences 6, World Scientific Publishing, Singapore, 2007.

8. T. Gutzmer and A. Iske: Detection of discontinuities in scattered data approximation. Numer. Algorithms 16, 1997, 155-170.

9. A. Harten, B. Engquist, S. Osher, and S. Chakravarthy: Uniformly high order essentially nonoscillatory schemes, III. J. Comput. Phys. 71, 1987, 231-303.

10. D. Hietel, K. Steiner, and J. Struckmeier: A finite-volume particle method for compressible flows. Math. Mod. Meth. Appl. Sci. 10(9), 2000, 1363-1382.

11. A. Iske: On the approximation order and numerical stability of local Lagrange interpolation by polyharmonic splines. In: Modern Developments in Multivariate Approximation, W. Haussmann, K. Jetter, M. Reimer, and J. Stöckler (eds.), International Series of Numerical Mathematics 145, 2003, Birkhäuser, Basel, 153-165.

12. A. Iske: Multiresolution Methods in Scattered Data Modelling. Lecture Notes in Computational Science and Engineering 37, Springer-Verlag, Berlin, 2004.

13. A. Iske: Polyharmonic spline reconstruction in adaptive particle flow simulation. In: Algorithms for Approximation, A. Iske and J. Levesley (eds.), Springer, Berlin, 2007, 83-102.

14. M. Junk: Do finite volume methods need a mesh? In: Meshfree Methods for Partial Differential Equations, M. Griebel, M.A. Schweitzer (eds.), Springer, 2003, 223-238.

15. M. Käser and A. Iske: ADER schemes on adaptive triangular meshes for scalar conservation laws. Journal of Computational Physics 205(2), 2005, 486-508.

16. R.L. LeVeque: Finite Volume Methods for Hyperbolic Problems. Cambridge University Press, Cambridge, UK, 2002.

17. X. Liu, S. Osher, and T. Chan: Weighted essentially non-oscillatory schemes. J. Comput. Phys. 115, 1994, 200-212.

18. F.J. Narcowich and J.D. Ward: Norm estimates for the inverses of a general class of scattereddata radial-function interpolation matrices. J. Approx. Theory 69, 1992, 84-109.

19. F.P. Preparata and M.I. Shamos: Computational Geometry. Springer, New York, 1988.

20. R. Schaback: Stability of radial basis function interpolants. In: Approximation Theory X: Wavelets, Splines, and Applications, C.K. Chui, L.L. Schumaker, and J. Stöckler (eds.), Vanderbilt Univ. Press, Nashville, 2002, 433-440.

21. R. Schaback: Error estimates and condition numbers for radial basis function interpolation. Advances in Comp. Math. 3, 1995, 251-264.

22. V.A. Titarev and E.F. Toro: ADER: arbitrary high order Godunov approach. J. Sci. Comput. 17, 2002, 609-618.

23. E.F. Toro, R.C. Millington, and L.A.M. Nejad: Towards very high order Godunov schemes. In: Godunov Methods (Oxford, 1999), Kluwer/Plenum, New York, 2001, 907-940.

24. H. Wendland: Scattered Data Approximation. Cambridge Univ. Press, Cambridge, UK, 2005. 